\title{
Transport of Electrons in Donor-Doped Silicon at Any Degree of Degeneracy of Electron Gas
}

\author{
Vilius Palenskis \\ Physics Faculty, Vilnius University, Vilnius, Lithuania \\ Email: vilius.palenskis@ff.vu.It
}

Received 8 May 2014; revised 14 June 2014; accepted 2 July 2014

Copyright (C) 2014 by author and Scientific Research Publishing Inc.

This work is licensed under the Creative Commons Attribution International License (CC BY).

http://creativecommons.org/licenses/by/4.0/

(c) (i) Open Access

\section{Abstract}

The general expressions, based on the Fermi distribution of the free electrons, are applied for calculation of the kinetic coefficients in donor-doped silicon at arbitrary degree of the degeneracy of electron gas under equilibrium conditions. The classical statistics lead to large errors in estimation of the transport parameters for the materials where Fermi level is located high above the conduction band edge unless the effective density of randomly moving electrons is introduced. The obtained results for the diffusion coefficient and drift mobility are discussed together with practical approximations applicable for non-degenerate electron gas and materials with arbitrary degree of degeneracy. In particular, the drift mobility of randomly moving electrons is found to depend on the degree of degeneracy and can exceed the Hall mobility considerably. When the effective density is introduced, the traditional Einstein relation between the diffusion coefficient and the drift mobility of randomly moving electrons is conserved at any level of degeneracy. The main conclusions and formulae can be applicable for holes in acceptor-doped silicon as well.

\section{Keywords}

Donor-Doped Silicon, Electrical Conductivity, Thermal Noise, Einstein Relation, Diffusion Coefficient, Drift Mobility, Hall Mobility

\section{Introduction}

Silicon is the main material for electronics during the last fifty years. Its basic parameters have been intensively treated theoretically and experimentally [1]-[18]. Diffusion coefficient and drift mobility are necessary for characterization of material quality and electronic transport properties. In spite of the importance of these 
quantities for device applications, such as bipolar transistors, the results on heavily doped regions are quite limited, while accurate values of electron mobility and diffusion coefficient are essential for advanced engineering. The theoretic problem for silicon and gallium arsenide usually has been treated through the Monte Carlo simulation [2]-[4]. The ratio of the classical value of the diffusion coefficient over the mobility for the majority- and minority-carriers satisfies the Einstein relation and equals $k T / e$ where $k$ is the Boltzmann constant, $T$ is the absolute temperature, and $e$ is the elementary charge [5]-[11]. Most published papers assume this relation to hold only for non-degenerate materials. Therefore, at thermal equilibrium, the diffusion coefficient $D$ of charge carriers in a degenerate semiconductor is often related to the mobility $\mu$ through the modified relation [2] [3] [5]-[8]:

$$
D=\frac{1}{e} \frac{n}{\mathrm{~d} n / \mathrm{d} E_{F}} \mu,
$$

where $n$ is the total density of free electrons in the conduction band and $E_{F}$ is the Fermi energy. Similar relation is used for holes. Equation (1) can be transformed into:

$$
e^{2} D \frac{\mathrm{d} n}{\mathrm{~d} E_{F}}=e n \mu
$$

where both sides mean the conductivity. But as it will be shown later the quantity $\mathrm{dn} / \mathrm{d} E_{F}$ in Equation (2) left side is proportional to the effective density of randomly moving electrons, while the expression en $\mu$ contains the total electron density $n$; this is wrong for degenerate silicon because the main contribution comes from the randomly moving electrons, located near the Fermi energy, while the contribution due to the electrons located deep below the Fermi level is next to zero because of limitations induced by the Pauli principle [19]-[21]. The randomly moving electrons not only determine the electric conduction and electron diffusion, but also electron heat capacity, electron thermal noise, electron heat conduction and other dissipative phenomena [22].

The electron Hall factor determined as the ratio of the Hall mobility over the drift mobility for non-degenerate semiconductors yields the values above unity [12] [13]. In some cases, the Hall factor of holes varies from 0.882 to 0.714 at $300 \mathrm{~K}$ over the acceptor density range $10^{14} \leq N_{A} \leq 3 \times 10^{18} \mathrm{~cm}^{-3}$ [14]. The factor tends to unity when the degenerate case is approached since the averaging of relaxation time $\tau$ over energy yields the value at the Fermi energy. In spite of huge amount of investigations, there are some unsolved problems of drift mobility and diffusion coefficient in silicon with high level of degeneracy of electron gas. We are going to illustrate that Equations (1) and (2) are not applicable for degenerate electron gas in silicon. Section 2 deals with the basic expressions. Section 3 presents and discusses the results of calculations for donor-doped silicon at any degree of degeneracy of electron gas.

\section{Basic Expressions of Electrical Conductivity Characteristics for Homogeneous Materials with One Type of Free Carriers at Any Degree of Degeneracy of Electron Gas}

The total density of the free electrons in the conduction-band is

$$
n=\int_{0}^{\infty} g(E) f(E) \mathrm{d} E
$$

where $E$ is the electron energy above the conduction band edge, $g(E)$ is the density of states (DOS) at the given energy, and $f(E)$ is the Fermi distribution function for electrons

$$
f(E)=\frac{1}{1+\exp (E-\eta) / k T},
$$

and $\eta$ is the chemical potential.

From the Fermi-Dirac statistics directly follows that the effective density of electrons $n_{\text {eff }}$ taking part in the random motion and, thus, in all kinetic processes depends not only on the DOS $g(E)$ of electrons and Fermi distribution function $f(E)$, but it depends also on the probability $f_{1}(E)=1-f(E)$ that any of such electron 
can leave the occupied energy level at a given temperature [19]-[21]:

$$
n_{\text {eff }}=\int_{0}^{\infty} g(E) f(E)[1-f(E)] \mathrm{d} E
$$

or

$$
n_{\text {eff }}=k T \int_{0}^{\infty} g(E)\left(-\frac{\partial f(E)}{\partial E}\right) \mathrm{d} E,
$$

because

$$
f(E) \cdot[1-f(E)]=-k T \frac{\partial f(E)}{\partial E} .
$$

The term $(-\partial f(E) / \partial E)$ represents the probability density of the randomly moving electrons:

$$
p(E)=-\frac{\partial f(E)}{\partial E}=f(E)[1-f(E)] / k T,
$$

and meets all requirements of the probability theory. Here it should be pointed that for materials with degenerate electron gas the total density of electrons $n$ is the fixed quantity, while the effective density of randomly moving electrons $n_{\text {eff }}$ is the stochastic variable. So, there are two parameters to characterize the free electron gas in metals and other materials with highly degenerate electron gas: the total density of the free electrons $n$ in conduction band and the effective density of randomly moving electrons $n_{\text {eff }}$.

Comparison of functions $f(E)$ and $f(E)[1-f(E)]$ for different normalized chemical potentials are shown in Figure 1. From this figure it seen that $f(E) \approx f(E)[1-f(E)]$, when $\eta^{*}=(\eta / k T)<-2$. In this case the classical statistics is applicable, and $n_{\text {eff }} \approx n$.

An approval of Equation (5) also follows from the general relation between the electrical conductivity $\sigma$ and the diffusion coefficient $D$ of electrons [22] [23]:

$$
\sigma=e^{2} D\left(\frac{\partial n}{\partial \eta}\right)_{T}
$$

After simple calculation of the derivative one obtains:

$$
\sigma=e^{2} D \int_{0}^{\infty} g(E) \frac{\partial f(E)}{\partial \eta} \mathrm{d} E=\frac{e^{2} D}{k T} \int_{0}^{\infty} g(E) f(E)[1-f(E)] \mathrm{d} E=\frac{e^{2} D}{k T} n_{\text {eff }},
$$

where expression

$$
n_{\text {eff }}=\int_{0}^{\infty} g(E) f(E)[1-f(E)] \mathrm{d} E
$$

is the same Equation (5) for the effective density of randomly moving electrons. Equation (10) unambiguously shows that the conductivity in all cases is determined by the effective density of randomly moving charge carriers determined through Equation (5) rather than the total free electron density in the conduction band. The total density $n$ can be used for determination of conductivity only for materials with non-degenerate electron gas.

Thus, the conductivity (Equation (10)) also can be presented as

$$
\sigma=\frac{e^{2} D}{k T} n_{\text {eff }}=e n_{\text {eff }} \mu_{\text {drift }},
$$

where $\mu_{\text {drift }}$ is the drift mobility of randomly moving charge carriers in the homogeneous materials with one type of the charge carriers (either electrons or holes). From this general relationship, which is applicable in all cases, one immediately gets the Einstein relation 


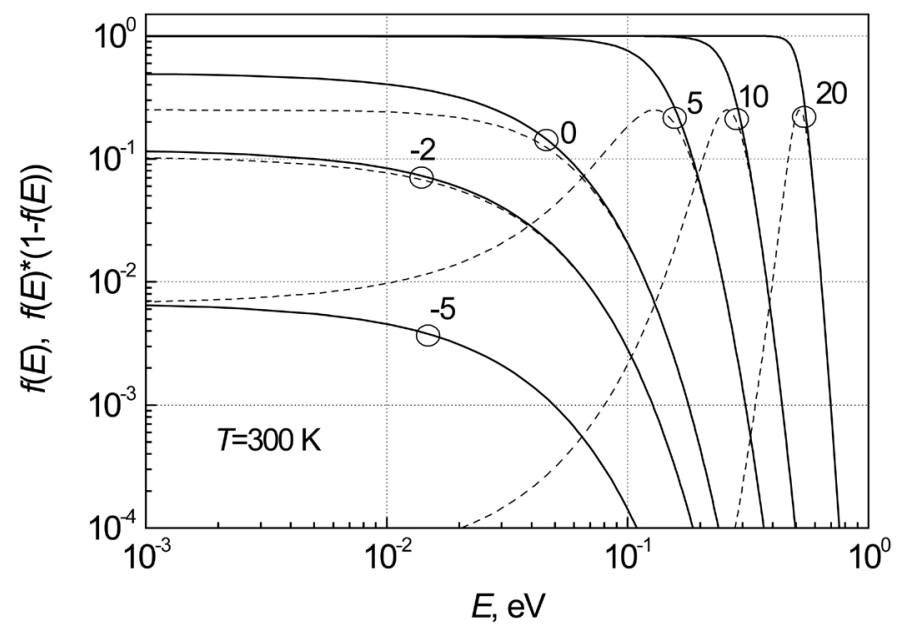

Figure 1. Comparison of functions $f(E)$ and $f(E)[1-f(E)]$ for different normalized chemical potentials $\eta^{*}=\eta / k T$. Solid lines are for $f(E)$, and dashed lines-for $f(E)[1-f(E)]$. The area under the curve $f(E)$ is proportional to the total density of free electrons in conduction band, and the area under the curve $f(E)[1-f(E)]$ - to the effective density of randomly moving electrons.

$$
\frac{D}{\mu_{\text {drift }}}=\frac{k T}{e} .
$$

In [20], accounting the concept of randomly moving charge carriers there were derived the following general relations, which are valid at any degree of degeneracy of electron gas in isotropic materials with one type of charge carriers:

For drift mobility:

$$
\mu_{d r i f t}=\frac{e\left\langle v^{2}\right\rangle\langle\tau\rangle}{3 k T}
$$

or

$$
\mu_{\text {drift }}=\frac{e\langle\tau\rangle}{m^{*}} \cdot \frac{\langle E\rangle}{(3 / 2) k T}=\frac{e\langle\tau\rangle}{m^{*}} \alpha_{\varepsilon} ;
$$

for electrical conductivity:

$$
\sigma=e n_{\text {eff }} \mu_{\text {drift }}=\frac{e^{2} D n_{\text {eff }}}{k T}=e n_{\text {eff }} \frac{e\langle\tau\rangle}{m^{*}} \cdot \alpha_{\varepsilon} ;
$$

for diffusion coefficient:

$$
D=\frac{1}{3}\left\langle v^{2}\right\rangle \cdot\langle\tau\rangle=\frac{k T \sigma}{e^{2} n_{e f f}}
$$

for drift velocity:

$$
v_{\text {drift }}=\mu_{\text {drift }} E=\frac{e\langle\tau\rangle}{m^{*}} \alpha_{\varepsilon} E ;
$$

where factor

$$
\alpha_{\varepsilon}=\frac{\langle E\rangle}{(3 / 2) k T}
$$


shows, how many times the mean kinetic energy $\langle E\rangle$ of randomly moving charge carrier is larger than $(3 / 2) k T$. The average kinetic energy of randomly moving electron $\langle E\rangle$ is defined by

$$
\langle E\rangle=\frac{\int_{0}^{\infty} E g(E) p(E) \mathrm{d} E}{\int_{0}^{\infty} g(E) p(E) \mathrm{d} E} .
$$

\section{Applications of the Basic Relations to Transport Features of Donor-Doped Silicon}

According to the above presented relations, one can evaluate the characteristics of donor-doped silicon with shallow donors (completely ionized at room temperature, $T=300 \mathrm{~K}$ ). Illustrations of Equations (3) and (5) for donor-doped silicon are presented in Figure 2. The effective density of randomly moving electrons $n_{\text {eff }}$ (shaded area) for highly degenerate material $\left(E_{F} / k T \geq 10\right)$ is many times lower than the total density of free electrons $n$ in the conduction band of silicon.

Here for calculation instead of chemical potential the Fermi level energy was used, because the difference between these quantities is about $0.01 \%$ even at room temperature [24].

Figure 3 presents the dependence of the Fermi energy $E_{F}$ and average kinetic energy $\langle E\rangle$ of randomly electrons on the total electron density $n$ in the conduction band of silicon. The Fermi energy was calculated from Equation (3), and $\langle E\rangle$ was obtained from Equation (19). The average kinetic energy of randomly moving electrons in $n S i$ for the total electron density $n$ smaller than $10^{18} \mathrm{~cm}^{-3}$ equals $\langle E\rangle=(3 / 2) k T$, but for $n>10^{20}$ $\mathrm{cm}^{-3}\langle E\rangle \approx E_{\mathrm{F}}$, and does not depend on temperature.

Comparison of the effective density of randomly moving electrons $n_{\text {eff }}$ with the total density of free electrons $n$ in conduction band of $n S i$ is presented for room temperature in Figure 4. Here

$$
\frac{n_{\text {eff }}}{n}=\frac{\int_{0}^{\infty} g(E) f(E)(1-f(E)) \mathrm{d} E}{\int_{0}^{\infty} g(E) f(E) \mathrm{d} E} .
$$

It illustrates that the effective density of randomly moving electrons $n_{\text {eff }}$ equals the total density of free electrons only when the shallow donor density $n_{D}$ is smaller than $10^{18} \mathrm{~cm}^{-3}$. However, a sharp decrease is found at high densities, and the ratio $n_{\text {eff }} / n$ is well below unity at $n \approx 10^{21} \mathrm{~cm}^{-3}$.

The effective density of randomly moving electrons $n_{\text {eff }}$ dependence on the total density $n$ of free electrons in $n S i$ at $T=300 \mathrm{~K}$ is shown in Figure 5. It shows that the effective density of randomly moving electrons $n_{\text {eff }}=n$ at $n \leq 10^{18} \mathrm{~cm}^{-3}$, but for $n>10^{20} \mathrm{~cm}^{-3} n_{\text {eff }} \sim n^{1 / 3}$.

The diffusion coefficient of randomly moving electrons in $n S i$ can be determined from Equation (16):

$$
D=k T /\left(e^{2} \rho n_{\text {eff }}\right) .
$$

The obtained results are presented in Figure 6. Here the resistivity $\rho$ dependence on the total free electron density in conduction band data have been taken from [25]-[28], and the effective density $n_{\text {eff }}$ of randomly moving electrons has been used from Figure 5.

The increase of the diffusion coefficient of randomly moving electrons at $n>10^{19} \mathrm{~cm}^{-3}$ is due to the increase of the average kinetic energy of the randomly moving electrons (Figure 3).

From Equation (15) follows that the drift mobility of randomly moving electrons can be obtained from the conductivity $\sigma$ or resistivity $\rho$ measurement results [25]-[28], when the dependence of the density of randomly moving electrons $n_{\text {eff }}$ on the total density of the free electrons (from Equation (3)) is taken into account:

$$
\mu_{\text {drift }}=\sigma /\left(e n_{\text {eff }}\right)=1 /\left(e \rho n_{\text {eff }}\right) .
$$

Comparison of drift and Hall [25] [26] mobilities for donor-doped silicon is presented in Figure 7. It is seen that the drift mobility of randomly moving electrons in silicon at high doping levels can be many times higher than the Hall mobility. 


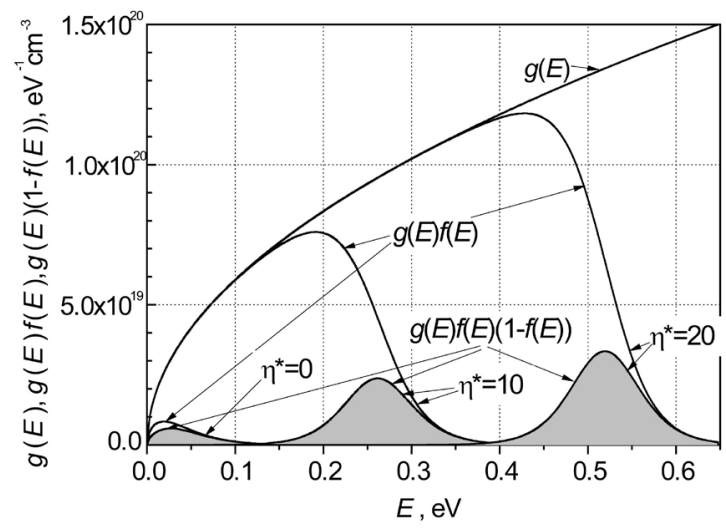

Figure 2. Illustration of energy dependent functions $g(E), g(E) f(E)$ and $g(E) f(E)(1-f(E))$ for donor-doped silicon at three normalized Fermi energy values $\eta^{*}=E_{F} / k T: 0,10$ and 20 at $T=300 \mathrm{~K}$. The area under the curve $g(E) f(E)$ represents the total density of free electrons $n$ in conduction band (Equation (3)), and the shaded area represents the effective density of randomly moving electrons $n_{\text {eff }}$ (Equation (5)). Here for $n S i \quad g(E)=\left(m_{d n} / \hbar^{3} \pi^{2}\right)\left(2 m_{d n} E\right)^{1 / 2}$ with $m_{d n}=1.08 m_{0}$.

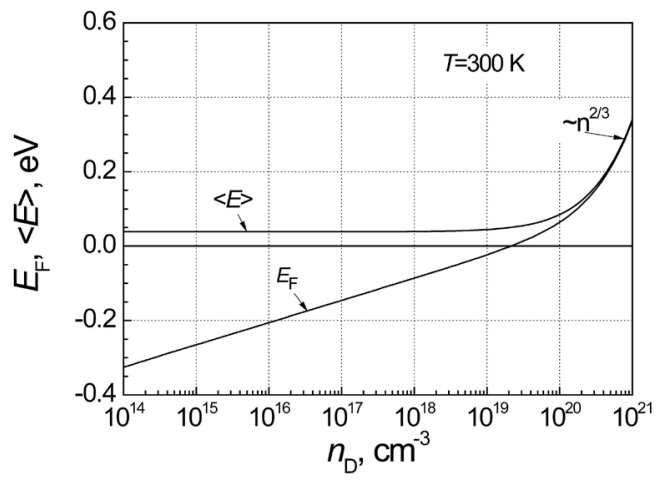

Figure 3. Fermi energy $E_{F}$ and average kinetic energy $\langle E\rangle$ of randomly moving electrons as a function of the total free electron density in $n S i$ at room temperature.

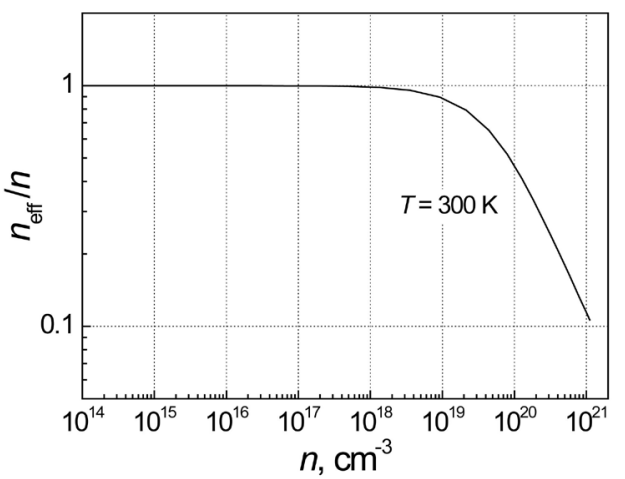

Figure 4. Ratio between the effective density of randomly moving electrons $n_{\text {eff }}$ and the total density of free electrons $n$ as a function of total density of free electrons in $n S i$ at room temperature. 


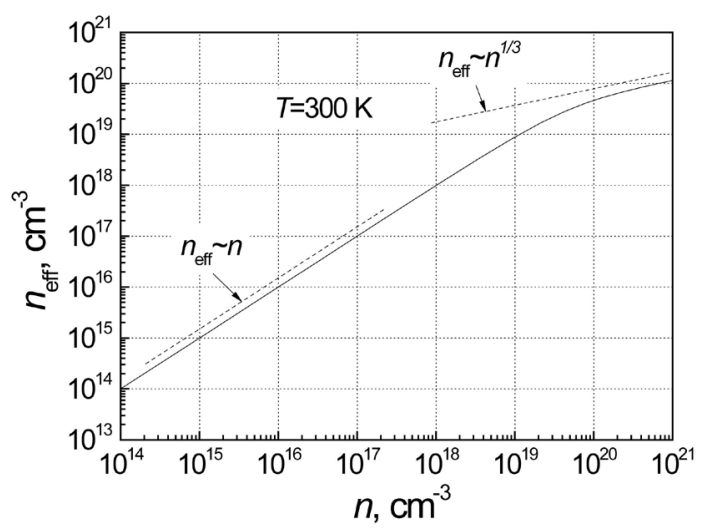

Figure 5. Dependence of the effective density of randomly moving electrons $n_{\text {eff }}$ on the total density $n$ of free electrons in $n S i$ at $T=300 \mathrm{~K}$.

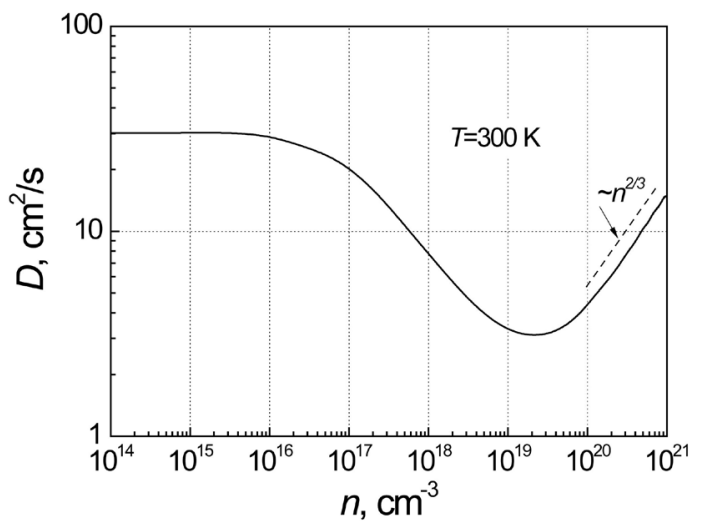

Figure 6. The diffusion coefficient of the randomly moving electrons dependence on the total density of the free electrons in the conduction band of $n S i$ at room temperature.

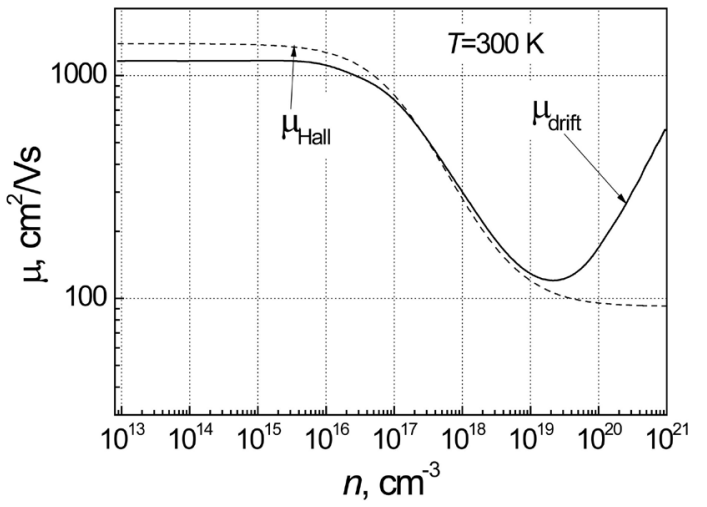

Figure 7. Hall $\mu_{\text {Hall }}$ (dash line, data from [25] [26]) and drift $\mu_{\text {drift }}$ (solid line) mobilities as functions of the total density of free electrons in $n S i$ at $T=300 \mathrm{~K}$.

The electron relaxation time at the Fermi level at room temperature is the same for all homogeneous metals and highly degenerate semiconductors and equals [29]:

$$
\tau_{F}=\hbar /(k T) ;
$$

where $\hbar=h / 2 \pi$ is the Plank constant. At highly degenerate $n S i$ the Hall mobility of electrons $\left|\mu_{H}\right| \approx 92$ 
$\mathrm{cm}^{2} / \mathrm{Vs}$ (Figure 7), it corresponds to $\left(\mathrm{m}^{*}=0.5 m_{0}\right)$, while the drift mobility can be many times higher, because it also depends on the kinetic energy of the randomly moving electrons. The relaxation time dependence on the total density of free electrons in $n S i$ at $T=300 \mathrm{~K}$, defined by using mobility data (Figure 7) and Equations (14) and (23) is presented in Figure 8. Here the effective mass of conduction of $n S i \quad m^{*}=0.26 m_{0}$ [25] [26] for $n \leq 10^{17} \mathrm{~cm}^{-3}$, and $\mathrm{m}^{*} \approx 0.5 \mathrm{~m}_{0}$ for $n>10^{19} \mathrm{~cm}^{-3}$. The relaxation time dependence on the total density of free electrons in conduction band can be approximated by such relation:

$$
\tau=\tau_{F}+1.5 \times 10^{-13} /\left(1+n / 1.5 \times 10^{17}\right) ;
$$

here $n$ is in $\mathrm{cm}^{-3}$.

a. c. conductivity usually is represented in the following form [24]:

$$
\sigma(\omega)=\frac{\sigma}{1-j \omega\langle\tau\rangle}
$$

and the relative permittivity for $(\omega\langle\tau\rangle) \gg 1$ as:

$$
\varepsilon_{r}=1-\frac{\omega_{p}^{2}}{\omega(\omega+j /\langle\tau\rangle)} \approx 1-\frac{\omega_{p}^{2}}{\omega^{2}} ;
$$

where

$$
\omega_{p}^{2}=\frac{\sigma}{\varepsilon_{0}\langle\tau\rangle} .
$$

Here $\omega_{p}$ is the plasma frequency. Accounting the basic Equation (15) for d. c. conductivity $\sigma$, the plasma frequency can be described as:

$$
\omega_{p}^{2}=\frac{e^{2}}{\varepsilon_{0} m^{*}} n_{e f f} \alpha_{\varepsilon} .
$$

The plasma frequency dependence on parameter $n_{\text {eff }} \alpha_{\varepsilon}$ for $n S i$ is shown in Figure 9. These results are near to that presented in [30].

The dielectric function (Equation (26)) vanishes at $\omega=\omega_{p}$, i.e. longitudinal vibrations (or the density oscillations of free electrons) of this frequency can appear in the electron gas even in absence of external excitations.

Usually the plasma frequency is presented in the following form [24] [31] [32]:

$$
\omega_{p}^{2}=\frac{e^{2} n}{\varepsilon_{0} m^{*}} .
$$

For silicon the density of states in conduction band is expressed as [24] [33]

$$
g(E)=\frac{m_{d n}}{\hbar^{3} \pi^{2}} \sqrt{2 m_{d n} E},
$$

$m_{d n}=1.08 m_{0}$ [32] is the effective mass of the density of states. In this case $n=n_{\text {eff }} \alpha_{\varepsilon}$, for silicon, it is illustrated in Figure 10. As it is seen there is a good coincidence of digital values of these quantities.

Though both Equations (28) and (29) give the same digital value of the plasma frequency, but it does not mean that all free electrons at high doping levels take part in collective oscillations. As it was shown for drift velocity (Equation (17)), the effective force acting to randomly moving electron in materials with highly degenerate electron gas is $\alpha_{\varepsilon}$ times higher than in non-degenerate case. Equation (28) is valid only then, when the average kinetic energy of electron $\langle E\rangle=(3 / 2) k T$. Coincidences of digital results from Equations (28) and (29) for highly degenerate materials are only then, when the Fermi surface is spherical or the density of states is parabolic. For materials with composite density of states (with non-spherical Fermi surfaces) Equation (29) is not valid [24] [34]. In principle, the expression (29) is misleading, because from it follows that all free electrons at high degenerate of electron gas take part in plasma oscillations. But this is not true, because the applied external electric field can only change the energy of randomly moving electrons, which energy is located near to the Fermi energy, while the contribution due to the electrons located deep below the Fermi level 


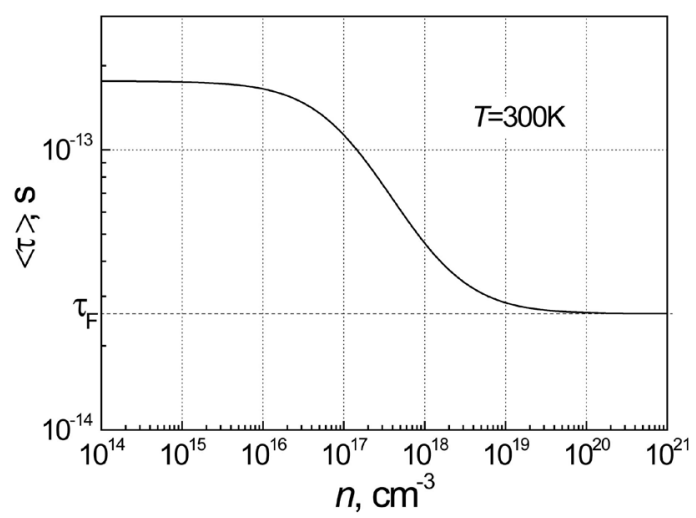

Figure 8. Relaxation time dependence on total density of free electrons in $n S i$ at $T=300$ K. $\tau_{F} \approx 2.6 \times 10^{-14} \mathrm{~s}$.

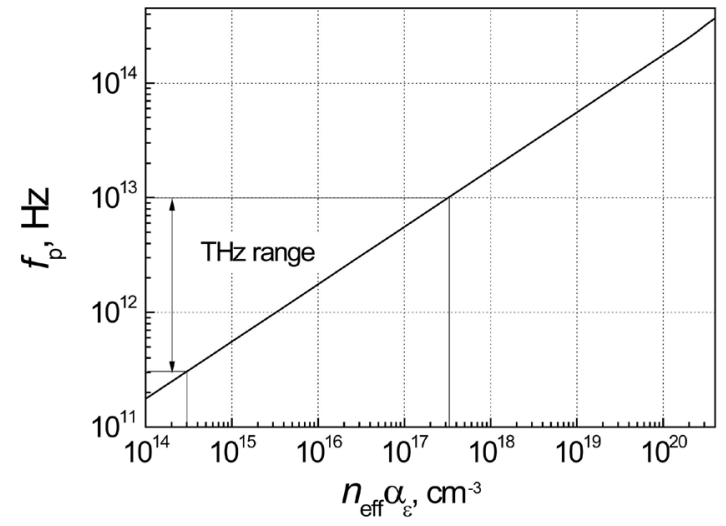

Figure 9. The plasma frequency dependence on parameter $n_{e f f} \alpha_{\varepsilon}$ for $n S i$.

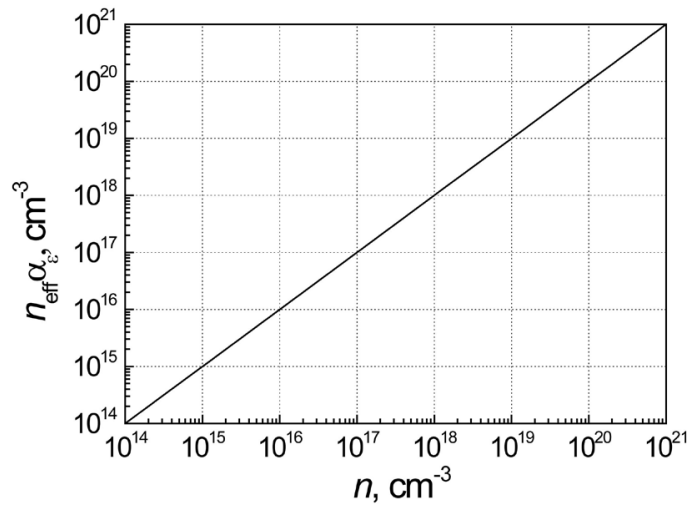

Figure 10. The parameter $n_{\text {eff }} \alpha_{\varepsilon}$ dependence on the total density $n$ of free electrons in $n S i$.

is near to zero due to limitations of the Pauli principle.

Terahertz (THz) plasma frequency range (see Figure 9) of silicon is very important for science and technology [32] [35]-[38]. In [31] [39], it is shown that reflectivity of electromagnetic waves has a minimum near to plasma frequency, and there the absorption by free charge carriers is increased [40]. This effect is used for THz radiation detection and fabrication of $\mathrm{THz}$ imaging cameras with plasma-wave-based silicon MOSFET detectors [41] [42]. 


\section{Conclusions}

This work tries to attract attention to interpretation of transport properties of degenerate semiconductors and to possible large errors caused when classical statistics is applied for estimation of transport parameters for heavily doped silicon. The general expressions for effective density of randomly moving electrons, their diffusion coefficient, drift mobility, and conductivity are presented and illustrated for electrons in silicon. The calculated values of effective density of randomly moving electrons, their diffusion coefficient, drift mobility, and plasma frequency for donor-doped silicon at any degree of degeneracy there are presented at room temperature. The comparison between the hall mobility and the drift mobility of randomly moving electrons is made and their specific behavior at high level of degeneracy is explained. The presented general expressions are valid for holes as well.

It is unambiguously shown that under equilibrium conditions the Nyquist formula, and the Einstein relation are always fulfilled for one type of charge carriers in homogeneous materials. The form of relation does not depend on the non-parabolicity of conduction band or on mechanism of scattering of charge carriers: The expressions of the Nyquist formula and the Einstein relation remain the same.

\section{Acknowledgements}

This study was funded by European Social Fund under Global Grant measure No. VP1-3.1-ŠMM-07-03-040.

\section{References}

[1] Ludwig, G.W. and Wakters, R.L. (1956) Drift and Conductivity Mobility in Silicon. Physical Review, 101, $1699-1701$. http://dx.doi.org/10.1103/PhysRev.101.1699

[2] Borovik, P. and Thobel, J.L. (1999) Monte Carlo Calculation of Diffusion Coefficients in Degenerate Bulk GaAs. Semiconductor Science and Technology, 14, 450-453. http://dx.doi.org/10.1088/0268-1242/14/5/014

[3] Thobel, J.L., Sleiman, A. and Fauquembergue, R. (1997) Determination of Diffusion Coefficients in Degenerate Electron Gas Using Monte Carlo Simulation. Journal of Applied Physics, 82, 1220-1226. http://dx.doi.org/10.1063/1.365892

[4] Kaiblinger-Grujin, G., Kosina, H. and Selberherr, S. (1997) Monte Carlo Simulation of Electron Transport in Doped Silicon. IEEEXplore, 444-449. http://dx.doi.org/10.1109/HPC.1997.592188

[5] Xiao, Z.-X. and Wei, T.-L. (1997) Modification of Einstein Equation of Majority- and Minority-Carriers with Band Gap Narrowing Effect in n-Type Degenerate Silicon with Degenerate Approximation and with Non-Parabolic Energy Bands. IEEE Transaction on Electron Devices, 44, 913-914. http://dx.doi.org/10.1109/16.568061

[6] Ristić, S.D. (1979) An Approximation of the Einstein Relation for Heavily Doped Silicon. Physica Status Solidi (a), 52, K129-K132. http://dx.doi.org/10.1002/pssa.2210520250

[7] Van Overstraeten, R.J., DeMan, H.J. and Mertens, R.P. (1973) Transport Equations in Heavily Doped Silicon. IEEE Transaction on Electron Devices, 20, 290-298. http://dx.doi.org/10.1109/T-ED.1973.17642

[8] Jain, R.K. (1977) Calculation of the Fermi Level, Minority Carrier Concentration, Effective Intrinsic Concentration, and Einstein Relation in n- and p-Type Germanium and Silicon. Physica Status Solidi (a), 42, 221-226. http://dx.doi.org/10.1002/pssa.2210420123

[9] Ghatak, K.P. and Mondal, M. (1992) The Diffusivity-Mobility Relation Innonparabolic Materials. Journal of Applied Physics, 71, 1277-1283. http://dx.doi.org/10.1063/1.351244

[10] Chakravarti, A.N. and Nag, B.N. (1974) Generalized Eistein Relation for Degenerate Semiconductors Having Nonparabolic Energy Bands. International Journal of Electronics, 37, 281-284. http://dx.doi.org/10.1080/00207217408900521

[11] Mohammad, S.N. and Bemis, A.V. (1992) The Einstein Relation for Degenerate Semiconductors with Nonuniform Band Structures. IEEE Transaction on Electron Devices, 39, 2826-2828. http://dx.doi.org/10.1109/16.168739

[12] Backenstoss, G. (1957) Conductivity Mobilities of Electrons and Holes in Heavily Doped Silicon. Physical Review, 108, 1416-1419. http://dx.doi.org/10.1103/PhysRev.108.1416

[13] Putley, E.H. and Mitchell, W.H. (1958) The Electrical Conductivity and Hall Effect of Silicon. Proceedings of Physical Society, 72, 193-200. http://dx.doi.org/10.1088/0370-1328/72/2/303

[14] Lin, J.F., Li, S.S., Linare, L.C. and Teng, K.W. (1981) Theoretical Analysis of Hall Factor and Hall Mobility in p-Type Silicon. Solid-State Electronics, 24, 827-833. http://dx.doi.org/10.1016/0038-1101(81)90098-8

[15] Bennett, H. (1983) Hole and Electron Mobilities in Heavily Doped Silicon: Comparison of Theory and Experiment. Solid-State Electronics, 26, 1157-1166. http://dx.doi.org/10.1016/0038-1101(83)90143-0

[16] Bennett, H. and Lowney, J. (1992) Calculated Majority- and Minority-Carrier Mobilities in Heavily Doped Silicon and 
Comparison with Experiment. Journal of Applied Physics, 71, 2285-2296. http://dx.doi.org/10.1063/1.351128

[17] Dziewior, J. and Silber, D. (1979) Minority-Carrier Diffusion Coefficients in Highly Doped Silicon. Applied Physics Letters, 35, 170-172. http://dx.doi.org/10.1063/1.91024

[18] Neugroschel, A. (1985) Minority-Carrier Diffusion Coefficients and Mobilities in Silicon. IEEE Electron Devices Letters, 6, 425-427. http://dx.doi.org/10.1109/EDL.1985.26178

[19] Palenskis, V., Juškevičius, A. and Laucius, A. (1985) Mobility of Charge Carriers in Degenerate Materials. Lithuanian Journal of Physics, 25, 125-132.

[20] Palenskis, V. (2013) Drift Mobility, Diffusion Coefficient of Randomly Moving Charge Carriers in Metals and Other Materials with Degenerated Electron Gas. World Journal of Condensed Matter Physics, 3, 73-81. http://dx.doi.org/10.4236/wjcmp.2013.31013

[21] Palenskis, V. (2014) The Effective Density of Randomly Moving Electrons and Related Characteristics of Materials with Degenerate Electron Gas. AIP Advances, 4, Article ID: 047119.

[22] Bonch-Bruevitch, V.L. and Kalashnikov, S.G. (1990) The Physics of Semiconductors. Nauka Press, Moscow.

[23] Bisquert, J. (2008) Interpretation of Electron Diffusion Coefficient in Organic and Inorganic Semiconductors with Broad Distributions of States. Physical Chemistry Chemical Physics, 10, 3175-3194. http://dx.doi.org/10.1039/b719943k

[24] Ashcroft, N. and Mermin, W.N.D. (1976) Solid State Physics. Holt, Rinehart and Winston, New York.

[25] Dargys, A. and Kundrotas, J. (1994) Handbook on Physical Properties of Ge, Si, GaAs and InP. Science and Encyclopedia Publishers, Vilnius.

[26] Jacoboni, C., Canali, C., Ottaviani, G. and Quaranta, A.A. (1977) A Review of Some Charge Transport Properties of Silicon. Solid-State Electronics, 20, 77-89. http://dx.doi.org/10.1016/0038-1101(77)90054-5

[27] Sze, S.M. (1983) VLSI Technology. McGraw-Hill, New York.

[28] INSPEC (1998) Properties of Silicon, EMIS Data Series No. 4. INSPEC, The Institute of Electrical Engineering, London.

[29] Devillers, M.A.C. (1984) Lifetime of Electrons in Metals at Room Temperature. Solid State Communications, 49, 1019-1022. http://dx.doi.org/10.1016/0038-1098(84)90413-7

[30] Arai, T. (1964) Plasma Oscillations in Heavily Doped n-Type Silicon. Proceedings of Physical Society, 84, 25-30. http://dx.doi.org/10.1088/0370-1328/84/1/305

[31] Seeger, K. (1973) Semiconductor Physics. Springer-Verlag, Wien.

[32] Saeedkia, D. (2013) Handbook of Terahertz Technology for Imaging, Sensing and Communications. Woodhead Publishing Ltd., Cambridge. http://dx.doi.org/10.1533/9780857096494

[33] Shalimova, K.V. (1985) Physics of Semiconductors. Energoatomizdat, Moscow.

[34] Oral, M., Long, L.L., Bell, R.J., Bell, S.E., Bell, R.R., Alexander, R.W. and Ward, C.A. (1983) Optical Properties of the Metals Al, Co, Cu, Au, Fe, Pb, Ni, Pd, Pt, Ag, Ti, and W in the Infrared and Far Infrared. Applied Optics, 22, 1099-1120. http://dx.doi.org/10.1364/AO.22.001099

[35] Lee, Y.S. (2009) Principles of Terahertz Science and Technology. Springer Science + Business Media, LLC, Berlin.

[36] Perenzoni, M. and Paul, D.J. (2014) Physics and Applications of Terahertz Radiation. Springer, Dordrecht.

[37] Brown, E.R. (2003) Fundamentals of Terrestrial Millimeter-Wave and THz Remote Sensing. International Journal of High Speed Electronics and Systems, 13, 995-1098. http://dx.doi.org/10.1142/S0129156403002125

[38] Shahzad, M., Medhi, G., Peale, R.E., Buchwald, W.R., Cleary, J.W., Soref, R., Boreman, G.D. and Edwards, O. (2011) Infrared Surface Plasmons on Heavily Doped Silicon. Journal of Applied Physics, 110, Article ID: 123105. http://dx.doi.org/10.1063/1.3672738

[39] Spitzer, W. and Fan, H.Y. (1957) Determination of Optical Constants and Carrier Effective Mass of Semiconductors. Physical Review, 106, 882-890. http://dx.doi.org/10.1103/PhysRev.106.882

[40] van Exter, M. and Grishkowsky, D. (1990) Optical and Electronic Properties of Doped Silicon from 0.1 to 2 THz. Applied Physics Letters, 56, 1694-1696. http://dx.doi.org/10.1063/1.103120

[41] Boppel, S., Lisauskas, A. and Roskos, H.G. (2013) Terahertz Array Imagers: Towards the Implementation of Terahertz Cameras with Plasma-Wave-Based Silicon MOSFET Detectors. In: Saeedkia, D., Ed., Handbook of Terahertz Technology for Imaging, Sensing and Communications, Chapter 8, Woodhead Publishing Ltd., Cambridge, 231-271. http://dx.doi.org/10.1533/9780857096494.2.231

[42] Lisauskas, A., Boppel, S., Matukas, J., Palenskis, V., Minkevičius, L., Valušis, G., Haring-Bolivar, P. and Roskos, H.G. (2013) Terahertz Responsivity and Low-Frequency Noise in Biased Silicon Field-Effect Transistors. Applied Physics Letters, 102, Article ID: 153505. http://dx.doi.org/10.1063/1.4802208 
Scientific Research Publishing (SCIRP) is one of the largest Open Access journal publishers. It is currently publishing more than 200 open access, online, peer-reviewed journals covering a wide range of academic disciplines. SCIRP serves the worldwide academic communities and contributes to the progress and application of science with its publication.

Other selected journals from SCIRP are listed as below. Submit your manuscript to us via either submit@scirp.org or Online Submission Portal.
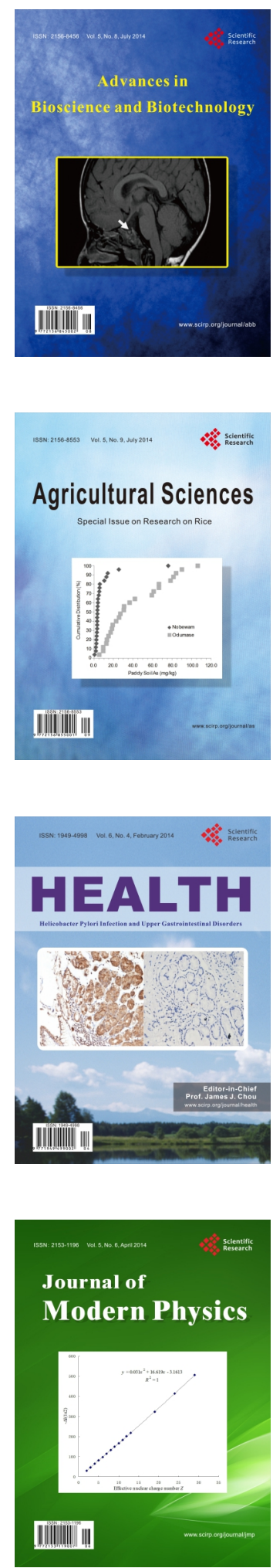
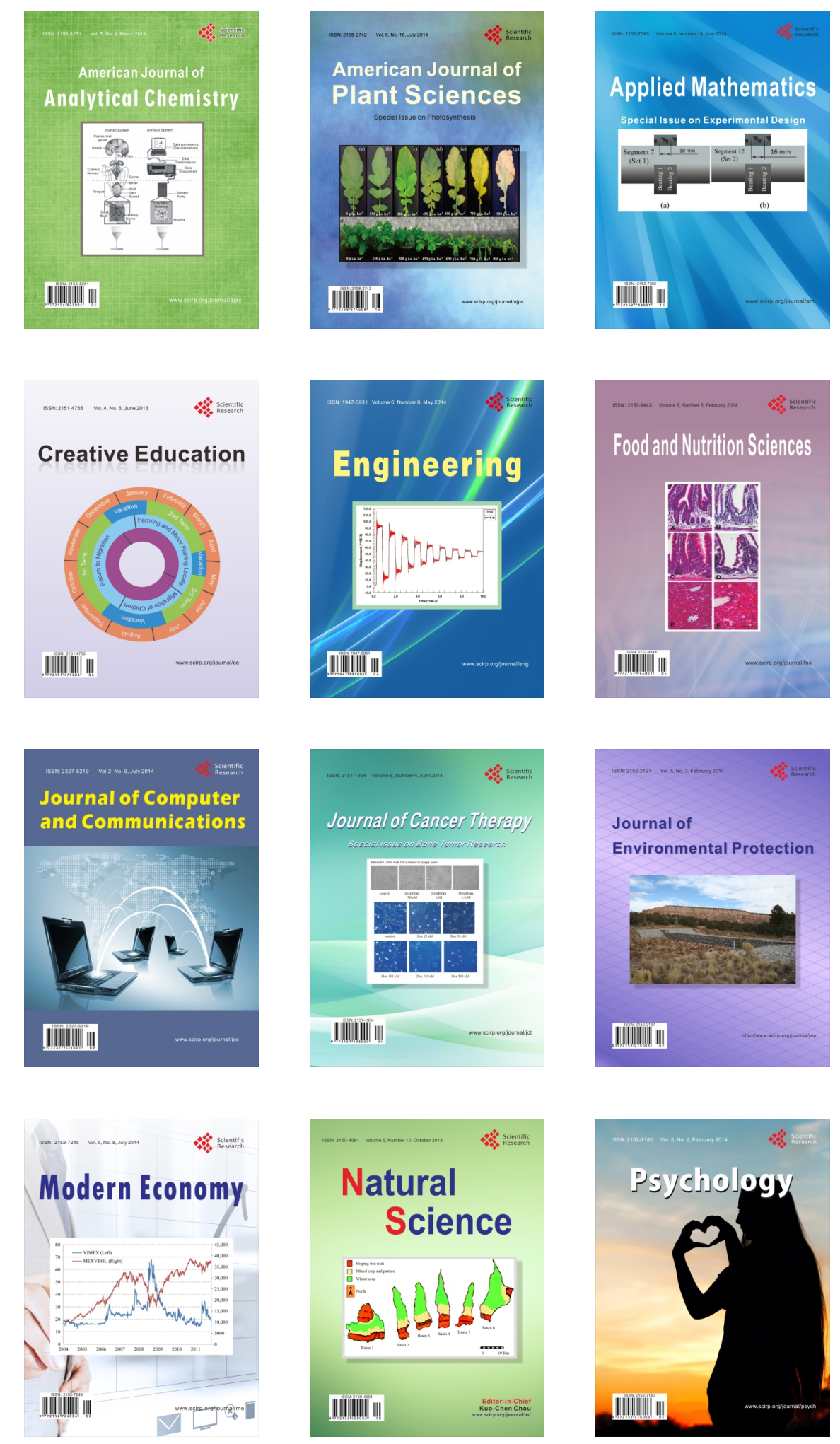